\title{
Transmedial Worlds with Social Impact: Exploring Intergenerational Learning in Collaborative Video Game Design
}

\author{
Juan C. Mansilla, Liliana Mejía Betancur, and Claudia Queiroz Lambach
}

\begin{abstract}
Juan C. Mansilla holds a PhD in information and communication sciences from the Sorbonne Paris Cité University. He is a member of the research laboratory Cultural Industries and Artistic Creation-LabEx ICCA (France) and, since 2017, has been a research associate at House of Social Sciences-Paris Nord. He has been a visiting scholar in São Paulo, Berkeley, Vancouver, Toronto, and Seoul. His research interests include media ecologies, urban sociology, and intergenerational programming. He is cofounder of Human Mount Institute an international network of social sciences researchers. Email: juan.mansilla@humanmount.com
\end{abstract}

Liliana Mejía Betancur holds a PhD in information and communication sciences from the Sorbonne Paris Cité University and a master's in culture and communication with a specialty in cultural and creative industries, media, web, arts from the University of Paris. She has been a social-journalist at the University of Antioquia (Colombia) and is a member of the research laboratory Cultural Industries and Artistic Creation-LabEx ICCA (France). Email: lilimejia95@gmail.com

Claudia Queiroz Lambach holds a PhD in information and communication sciences from the Sorbonne Paris Cité University and a master's in theory of visual culture from IADE-Faculty of Design Technology and Communication, European University, Portugal. She is a specialist in the history of modern and contemporary art and a member of the research laboratory Cultural Industries and Artistic Creation-LabEx ICCA (France). Email: cmql@hotmail.com

This article's focus is the creation of intergenerational game design experiences supported by phygital video games (i.e., games that feature both digital and physical elements) for children 8-12 years of age and elders 65 years and over living in Paris and Bagnolet, France. The purpose of the article is twofold: first, to give an account of our protocol activity to highlight the implementation difficulties associated with this kind of intergenerational gameplay, and second, to inspire researchers, educators, and caregivers to include innovative uses of transmedia world building and collaborative game design as tools to connect different generations and foster community participation in intergenerational programs.

Key words: children; elders; intergenerational game design; phygital gameplay; transmedia world building

technologies (ICTs). We evaluated these topics according to the participants' behaviour during the workshops, focusing on implicit and explicit expressions of solidarity between participants and the creative production of video games based on the affordances of narratives and game design.

\section{The Intergenerational Creative Memory and Multimedia Conception (COMMIC) project}

In 2016, a multidisciplinary team of researchers in educational anthropology, information and communication sciences, and visual studies created research laboratory Cultural Industries and Artistic Creation-LabEx ICCA (France) and implemented in two French cities, Paris and Bagnolet (http://projectcommic. $\mathrm{com} /$ ). The project consisted of the implementation of several workshops where children from 8-12 years of age and elders 65 years and over designed a video game using Bloxels Builder (BB), a tablet computing application based on the identification of colour and spatial position of small plastic cubes on a big plastic board game. Using a quasi-experimental methodology, the purpose of the study was to understand three emergent topics: (1) the intergenerational transfer of explicit and implicit knowledge; (2) the intergenerational learning associated with transmedia narrative design; and (3) the generational uses of information and communication COMMIC, a two-year research project funded by the 
The COMMIC social approach aimed at bridging the "age segregation" of classical institutions (e.g., elementary schools, retirement homes, children's recreation centres, senior clubs) in the local communities of these two cities. We were aware of the social and scientific dimensions of the project, as well as the empowering experience of intergenerational activities for participants (Newman \& Smith, 1997; Zhang, Schell, Kaufman, Salgado, \& Jeremic, 2017). We built spaces of intergenerational exchange to facilitate the transfer, not only of digital know-how, but also of social, empathic, and problem-solving skills. Further, learning from phygital video games (i.e., games that exploit both digital and physical elements) allows participants to develop creative thinking, procedural memory, spatial logic, and narrative design (Kaufman, Sauvé, Renaud, Sixsmith, \& Mortenson, 2016).

We conducted six workshops in three different kinds of institutions for elders: an accommodation establishment for dependent elders (établissement d'hébergement pour personnes âgées dépendantes), where seniors live and are assisted by people with medical training; a seniors' club (club des seniors), where seniors participate in different leisure activities on a drop-in basis (e.g., card games, cooking classes); and an autonomous retirement home (foyer-logement pour personnes âgées), where seniors live without medical assistance. Educators bring children from elementary schools and recreational centres to the institutions. These visits were introduced as part of the COMMIC project, which brings an added social and local community dimension to our program. In total, 52 children and 47 elders participated in the COMMIC project. Children were students from different elementary schools in Paris and Bagnolet, and elders came from different kinds of seniors' institutions in the same cities, as explained above. Between 8 and 12 children and 6 and 10 elders were present in each workshop. Participants were divided into three intergenerational teams during each workshop; therefore, every team had the same number of participants. However, the ratio of children to elders was not the same in every workshop, which elicited different results from one workshop to another, as stated in the results section.

Every workshop was carried out in three sequential phases: (1) construction of video games (creativity phase); (2) deconstruction of created games (memory phase); and (3) playability of video games (gameplay phase). The three intergenerational teams in every workshop performed each of the phases, at the same time. In the creativity phase, participants were expected to place small coloured cubes on a plastic board, and then, using the BB application, turn it into a playable level game by taking a picture of it. A playable level game has a narrative sequence (i.e., a fictional world with a character able to go from start to ending point). The BB application recognizes the colour and position of the cubes on the board and transforms them into gameplay objects (e.g., the yellow cubes are coins), so characters collect them throughout the game. In the memory phase, participants have to rebuild the physical board game design of other teams. To this end, they play the digital game to identify the physical bases (i.e., cube's colours and spatial position) used in the narrative gameplay sequence. Finally, in the gameplay phase, participants are expected to test the playability of each video game production. Twenty-eight games were built during the six workshops; two of them were not playable (i.e., the main character was blocked at some stage of the game). All video game productions are saved on the Infinity Wall of the BB application, accessible in the application platform or on the website (http://home.bloxelsbuilder.com/index.html). The Infinity Wall uses an x/y-coordinated method to localize the published games. COMMIC games are in the x-coordinate from 103 to 130, with y-coordinate 50, (e.g., 110,50).

\section{A transmedial gaming world experience}

New media researchers Lisbeth Klastrup and Susana Tosca (2004) coined the term transmedial worlds to indicate "abstract content systems from which a repertoire of fictional stories can be actualized or derived across a variety of media forms" (p. 1). Participants in COMMIC used BB application affordances, a kind of "abstract content system," to produce different narrative discourses evolving in fictional worlds. The BB software developer 
introduces a predetermined number of game rules and fictional characters, that is, the application developer "meta-discourse" (Sánchez-Mesa, Aarseth, Pratten, \& Scolari, 2016, p. 13). However, users have the capacity to evolve in an autonomous way to create new gaming content derived from this predetermined discourse. Recently, the BB software developer has enriched this meta-discourse by introducing fictional references from Star Wars, which, in turn, increases the transmediality of the BB video games. Participants in COMMIC were exposed to multiple narrative discourses across a variety of media forms before, during, and after the hands-on activities. In the COMMIC approach, the game design starts with existing popular media references (e.g., cinema, arcade games), and then participants use mobile devices to create fictional gaming worlds. Finally, games are published online, where any user can expand the transmedial world and thereafter redefine the whole fictional space and so on.

In three of the six workshops, the participants received detailed instructions about the goal of the activity (i.e., to create a playable game), which implied different results from the three other workshops. We started these workshops by explaining the logic of a playable level game. We presented video footage of existing narrative discourses in popular media culture to illustrate the desired results. For this purpose, we two compared short films: Buster Keaton's ending sequence from Seven Chances (1925) and Super Mario Bros video games, published by the YouTuber Gameplaygag (https://goo.gl/ExDd6U). Children and elders easily understood these popular media references and their relation to the proposed game design goal. The participants then created narrative spaces where the stories would take place (i.e., transmedial worlds), rather than transmedia storytelling. This means that a COMMIC video game production is a world-building process with the necessary elements to articulate events and generate innumerable user-stories. The transmedia world-building strategy in intergenerational learning allows participants to incorporate multiple cultural references in the same narrative sequence. This would both overcome generational gaps between "old" and "new" popular media culture and enhance generational empathy. This should not be considered a firm statement, but a useful strategy that, in our experience, allows us to continue with the planned activities. For example, children considered the Buster Keaton character as a hero, and seniors engaged with them by talking about other silent film heroes, such as Charlie Chaplin. We observed fruitful conversations between the two groups when they agreed on popular media culture features. We called this approach transmedial gaming world with social impact.

\section{Digital content production technology in intergenerational learning}

Designing and creating gaming content using digital content production technology (DCPT) was one of the most important challenges participants faced. The collaborative design of a video game requires digital, creative, and collaborative skills. Participants need to understand the tools they have at their disposal to design the game. This implies knowledge of both the application meta-discourse and the technical possibilities of the DCPT. Participants in a phygital game should be aware of the possibilities of the physical world and of their consequences in the digital world. At the same time, participants need to translate their solutions into collaborative strategies, which are expressed through verbal and nonverbal communication. Intergenerational learning with DCPT is particularly sensitive to nonverbal interactions (Hock-Koon \& Mansilla, 2015). Indeed, some research suggests that the modes of expression vary according to age, and that children are more diverse in their methods of communication than are elder adults (Heydon \& O'Neill, 2014). Other important aspects to consider are the transformation of the human learning process later in life (e.g., declines in procedural memory; Bonder \& Wagner, 2001) and agerelated deficits such as movement coordination and reaction time (Lesnoff-Caravaglia, 2007). These differences raise questions, such as, how can implicit and explicit generational know-how be measured? How do we take into account these communicational, biological, and cultural generational gaps in collaborative environments? There is still a lot of ground to be explored in this field. Therefore, it seems crucial to understand how intergenerational 
collaborative experiences of transmedia production impact the existing generational gaps in our societies.

\section{Findings}

We used a protocol for observing and videorecording behaviour during the workshops (Derry et al., 2010) to identify the degree of participation, solidarity, and enjoyment of the participants. As explained before, in three of the six workshops, we decided to give the participants detailed instructions about the goal of the activity, while in the three other workshops the participants built by themselves the meaning of a "playable game." We took field notes and recorded videos, especially during the hands-on activities, which allowed us to have a better understanding of the participants' nonverbal communication. The participants' names are changed here for anonymity. In this paper, we have translated all quotations of participants from French into English. We added field notes and analysis after every quotation. We propose a detailed account of the main difficulties and lessons. They are not necessarily presented according to the three phases of the protocol as described before (i.e., the creativity phase, the memory phase, and the gameplay phase). However, this does not mean that there were phases without difficulties, but, as we will show, the creativity phase was the most challenging for both groups of participants. This may have been the case because they needed to interpret more information at the beginning of the activity. Some of our main findings were as follows.

Most elder participants in COMMIC were women. During the six workshops, according to our observations, the men seemed less willing to join the hands-on activities. Several times men said "I prefer to watch." An elder participant who had come to see the workshop set up left, saying, "This is not for me at all." The workshops took place in the early afternoon, coinciding with siesta time at the retirement homes, which could potentially demotivate some elderly residents. Other residents said they were "afraid" of new technology and not curious to try something new. In general, before the activity, children were eager to use the tablets, and elders seemed indifferent. We suggest creating the collaborative intergenerational teams (CIT) with a few participants in each group to promote senior participation (see lesson 1 at the end of the article). We can affirm that the intergenerational exchange is not entirely natural. Children do not instinctively invite elders to play. However, when asked, they are usually very patient, explaining to elder participants how to proceed. The following is an example of an elder participant whom we found to be above average in adopting new media technology.

Isabelle (86-year-old-woman) asked us, a little anxiously, "Do you think I would be able to do it? Because I am really a beginner." She was very motivated by the activity and engaged with the game. Children helped her to place the colour cubes on the plastic game board. Even if children did almost all the activity, the fact that Isabelle was at the centre of the table and the only elder in her team allowed an easy and effective intergenerational exchange among the team participants. She had a very good understanding of how the game worked, and she did not hesitate to interact with the children, ask them for information, or even give them advice. Isabelle, who told us after the workshop she had a computer in her room, was familiar with the new technologies and had completely enjoyed the game. This remark is important because she was the only participant in this retirement home to be so comfortable with new technologies. She was not afraid or overwhelmed by this kind of activity. She also took some pride in being able to play and understand the game. She affirmed throughout the interview that she had a good time interacting with the children.

Children wanted to have the tablet in their hands as long as possible, especially the boys. Frequently, boys did not let girls play too much with the tablet. We suggest some strategies to create a more balanced intergenerational experience. For example, we assign small tasks to each participant and avoid the simplistic division "children in charge of tablets and seniors in charge of physical objects" (see lesson 3 at the end of the article). In general, a 
leader appears in each intergenerational team (usually a child, but sometimes a senior) and takes charge of the game (e.g., he or she assigns tasks to other team members). In general, children explain to the elder participants the relationship between the colour of the cubes and their function, and "advise" them about the spatial position of the cubes on the plastic board game, as the following example shows.

Children tend to guide elders through the activity: “Turn around and shoot ... Take a life." One elder participant said, "But if you play this all day, you go crazy at night. You're used to making it, we're not." Another added: "I'm not capable of doing this!"

Children wanted to go fast with the plastic board game so they could move on to the digital gameplay stage. This behaviour inhibited the interaction of elder participants. However, children explained to elders the game rules with verbal and nonverbal communication, as the following example shows.

At first, Colette (82-year-old woman) did not want to play, on the pretext that "it's impossible!" A child explained to her, "You have to jump with B, shoot with A. You have to do it at the same time, use both hands." Another child said to her, "With this hand you press this button."

As we expected, some elders with disabilities living in care centres started to leave the activity at this point. Some of them argued that they were tired. Bonder and Wagner (2001) have argued that attention spans for people in this age group can be short, but we surmised that some of the elder participants did not really have a strong interest in the activity. Additionally, some disabilities like dementia and those affecting movement and coordination were more likely to make their participation difficult. The enjoyment of elders and the interest they showed in the activity can be seen in their observation of the younger people, which prompted childhood reminiscences, such as the one bellow, as well as in their participation in the workshop.

Colette said, "Just seeing them reminds me of school." And she started to tell me some of her childhood memories. Another elder participant said, "It's good; it's modern," while another senior participant said, "It's good to see the children like this, we never do that."

For younger people, according to our observations, their interest in the activity is more related to handling the new technologies. Both groups of participants demonstrated increased generational empathy, characterized by children offering support to elders and the latter engaging more with technological hands-on activities. For example, children gave explicit instructions to elder participants about how to control the characters in the games. In some cases, elders gave instructions to younger participants about the memory stage of the game. Both verbal and nonverbal communication were used in their interactions. This way, elder and child participants were able, not only to cooperate in a technological challenge using phygital resources, but also to know more about the individuals in their teams (their names, ages, classes in school, etc.).

The workshops, where we presented detailed instructions about the goal of the activity followed by video footage showing popular media examples of narrative sequences, allowed participants to carry out the activities in a more independent way. Nevertheless, when these initial instructions and references were absent, we observed more dialogue between the participants as they tried to figure out what exactly "a playable level game" meant. Some children engaged creatively with the game and wanted to insert traps, lives, enemies, and hazards while designing. They already seemed to be projecting the second part of the activity, that is, the memory phase, while elder participants were more focused on the current phase. This difference in knowledge caused some difficulties, particularly in the creativity phase, when the concept of playability was being established in each team. Children were in a competitive logic vis-à-vis the other teams without taking into account the main goal of the activity (i.e., to create a playable game). 
During the memory phase, the children continued to interact with the elder participants. All participants understood the colour functions of the cubes. An elder said, “There's purple here." "No, it's not purple, it's danger," replied a child. Exchanges worked. Very quickly, the children wanted to play with tablets while the elder participants talked among themselves about different topics, not necessarily about the workshop activity. Not all the games produced during the workshop were fully playable. This did not discourage the participants. On the contrary, nonplayable levels awakened in both children and elders more curiosity about why the game did not work properly. The intervention of researchers was necessary to fix the logic of spatial building to advance to the next proposed phase of the activity.

\section{Lessons learned}

Lesson 1: Less is more

Collaborative intergenerational teams (CITs) are more effective with few participants. A ratio of one child per elder is more desirable to carry out a specific task than a CIT of five or six participants (even if the ratio is 50/50). A good strategy is to divide the whole activity into sub-activities and the latter into minimal logic tasks. In this way, CITs of 2 (maximum 3) participants could be responsible for one goal activity. This approach will ensure more intergenerational interaction and more engaged participants.

\section{Lesson 2: The challenging "siesta time"}

It is more convenient for elders to participate in intergenerational activities in the morning or in the middle of the afternoon. The hour after lunch (siesta time) in retirement homes could provoke low motivation in the participants. However, the schedule of activities has to rely on the children's and educators' school schedules.

\section{Lesson 3: Controlling the appealing digital experience}

A phygital game activity is a promise of new technological and digital manipulation; children and elders are aware of that. They have preconceived ideas about ICT practices and digital experiences. Both children and elders are affected by the presence of DCPT. Children pay a lot of attention to the tablet computer manipulation, sometimes at the expense of the intergenerational interaction. When children are too focused on tablets, elder participants are more inhibited in their interactions with them. This might happen because some elders are not so familiar with this technology, at least not as familiar as the new generation. A simple strategy to avoid this distance is to create small intergenerational teams (see also Lesson 1) and assign each participant different tasks related to the tablet manipulation, not just children with tablets and elders with physical objects. To avoid a technological language gap between participants, it is necessary to design iterative and engaging activities for each participant across both physical and digital dimensions. For example, in our activity an intergenerational team was asked to create a game level. An elder participant on the team created the floor and the enemies by putting the coloured cubes on the physical board; a child created the enemies and the powers, and another child scanned the board with the tablet. In the next phase, the elder participant tested the level using the tablet and the child fixed the imperfect sides of the level. Small tasks and iterative engagement across physical and digital dimensions allowed a balanced interaction between participants: one of our research goals.

The intergenerational activities are first of all generational meetings where participants (re)discover "the others" and consequently themselves. Transmedia and intergenerational program (IP) practitioners can find in this article some cues to address the fundamental generational gaps in collective experiences involving the use of media technology. From our perspective, accepting the process as the most important social and research goal (instead 
of measurable results) gives us the opportunity to observe the meaning of cooperation and technological practices between children and elders. This process with social impact, new transmedia possibilities, and popular media culture references could be further used to bring together older and younger generations.
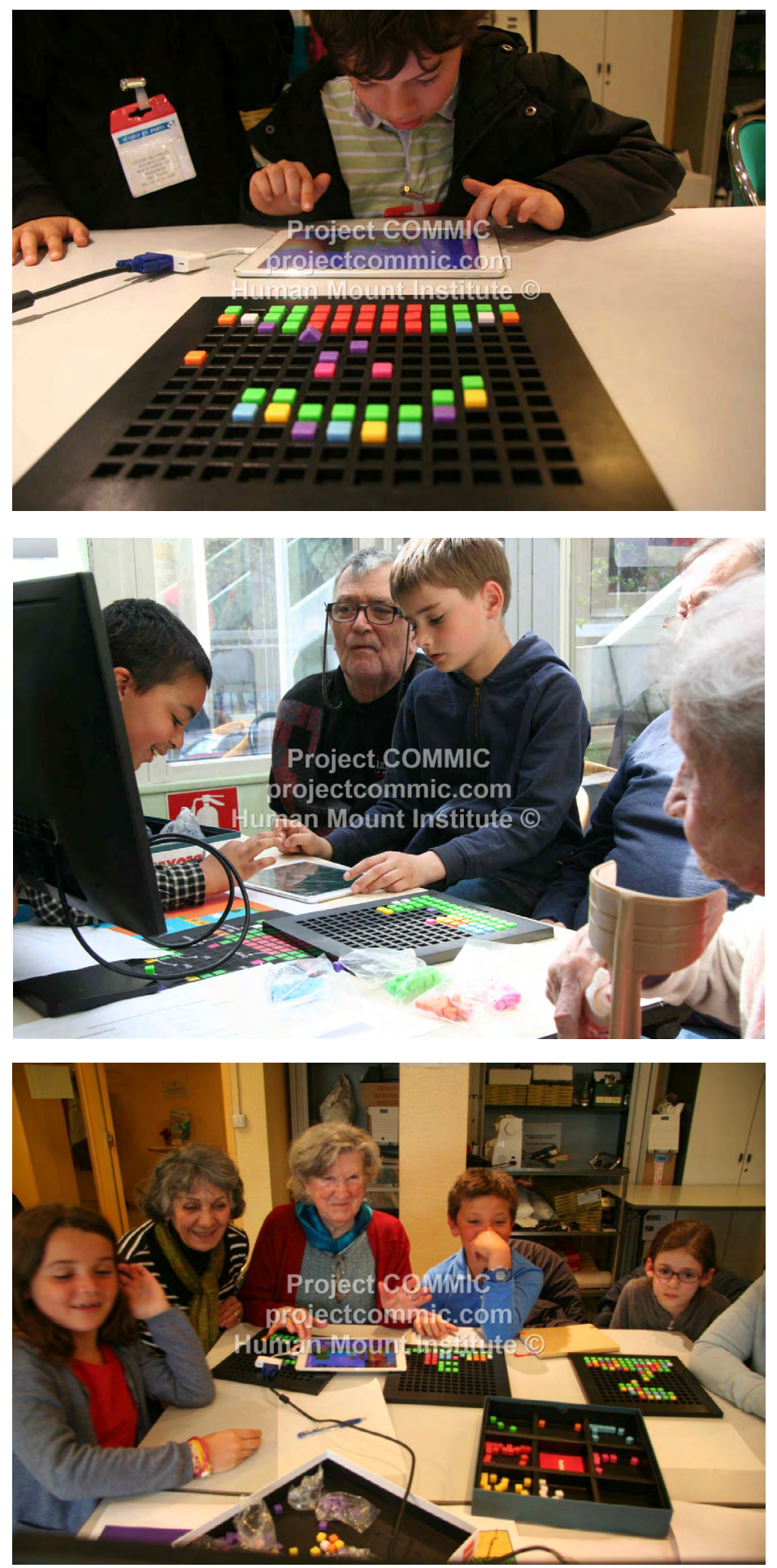

Project COMMIC: Intergenerational Learning in Collaborative Video Game Design. 


\section{Acknowledgements}

The COMMIC project received funds from LabEx ICCA, an interdisciplinary research laboratory centered around the arts, culture, and digital markets, financed by the Government of France through the program "Investissement d'Avenir" [Future Investment]. COMMIC was supported by the House of Social Sciences (Maison des Sciences de l'Homme, MSH Paris Nord) and by Pixel Press Technology, LLC.

\section{Endnotes}

1 In this approach, storytelling plays a subsidiary role in favour of characters and world building, unlike transmedia storytelling, which "proposes a common experience that encompasses various media and devices, all united by a narrative link" (Scolari, 2014, p. 71).

\section{References}

Bonder, B., \& Wagner, M. B. (2001). Functional performance in older adults. Philadelphia, PA: F. A. Davis.

Derry, S., Pea, R., Barron, B., Engle, R., Erickson, F., Goldman, R., Hall, R., Koschmann, T., Lemke, J., Sherin, M., \& Sherin, B. (2010). Conducting video research in the learning sciences: Guidance on selection, analysis, technology, and ethics. The Journal of The Learning Sciences, 19, 3-53. https://doi.org/10.1080/10508400903452884

Heydon, R., \& O’Neill, S. (2014). Songs in our hearts: The affordances and constraints of an intergenerational multimodal arts curriculum. International Journal of Education \& the Arts, 15(16). Retrieved from https://eric.ed.gov/?id=EJ1045920

Hock-Koon, S., \& Mansilla, J. C. (2015). Elderly people using tablets: Unexpected issues highlighted in an intergenerational activity (pp. 1092-1097). Paper presented at the Society for Information Technology \& Teacher Education International Conference, Association for the Advancement of Computing in Education, Las Vegas, Nevada, March 2, 2015. Retrieved from https://www. learntechlib.org/p/150142/

Kaufman, D., Sauvé, L., Renaud, L., Sixsmith, A., \& Mortenson, B. (2016). Older adults' digital gameplay: Patterns, benefits, and challenges. Simulation \& Gaming, 47(4), 465-489. https://doi.org/10.1177/1046878116645736

Klastrup, L., \& Tosca, S. (2004). Transmedial worlds: Rethinking cyberworld design. In 2004 International Conference on Cyberworlds (pp. 409-416). https://doi.org/10.1109/CW.2004.67

Lesnoff-Caravaglia, G. (2007). Health aspects of aging: The experience of growing old. Springfield, IL: Charles C. Thomas.

Newman, S., \& Smith, T. B. (1997). Developmental theories as the basis for intergenerational programs. In S. Newman \& T. B. Ward (Eds.), Intergenerational programs: Past, present, and future (p. 247). Washington, DC: Taylor \& Francis.

Sánchez-Mesa Martínez, D., Aarseth, E. J., Pratten, R., \& Scolari, C. A. (2016). Transmedia (storytelling?): A polyphonic critical review. Artnodes, 18. http://doi.org/10.7238/a.v0i18.3064

Scolari, C. A. (2014). Transmedia storytelling: New ways of communicating in the digital age. In Anuario AC/E de cultura digital-Focus 2014: The use of new technologies in the performing arts (pp. 68-79). Madrid, España: Acción Cultural Española. Retrieved from https://repositori.upf.edu/handle/10230/26242

Zhang, F., Schell, R., Kaufman, D., Salgado, G., \& Jeremic, J. (2017). Social interaction between older adults (80+) and younger people during intergenerational digital gameplay. In J. Zhou \& G. Salvendy (Eds.), Human aspects of IT for the aged population: Applications, services, and contexts (Vol. 10298, pp. 308-322). Cham, Switzerland: Springer International. https://doi. org/10.1007/978-3-319-58536-9_25 